\title{
La gouvernance de la santé animale : entre biosécurité et bien public mondial
}

\author{
Muriel Figuié* \\ Sociologie, CIRAD, UMR MoISA, Univ Montpellier, CIRAD, CIHEAM-IAMM, INRAE, Institut Agro, IRD, Montpellier, France
}

Reçu le 18 octobre 2018. Accepté le 18 mars 2021

Écrit avant la pandémie de Covid-19, cet article apporte un éclairage essentiel sur les formes de gouvernement et les relations entre savoir et pouvoir associées à l'émergence de la santé publique vétérinaire, et plus récemment à celle de la santé globale (associée au concept One Health) comme bien public mondial. L'enquête de terrain dont il est question ici permet de saisir les implications de ce nouveau cadre pour les éleveurs de volailles vietnamiens. Si une coordination internationale pour le contrôle des zoonoses est essentielle, les évolutions actuelles autour de la notion de biosécurité engendrent des dispositifs qui peinent à prendre en compte les pratiques et savoirs des acteurs de terrain, ainsi que les animaux en tant qu'êtres sensibles. Penser les apories de la santé animale comme bien public mondial peut ainsi contribuer à enrichir la réflexion sur les communs mondiaux, comme l'a suggéré le séminaire interdisciplinaire organisé à Montpellier par NSS-Dialogues en juin 2018, séminaire au cours duquel une première version de ce texte avait été présentée.

La Rédaction

\begin{abstract}
Résumé - L'article examine l'histoire, les définitions, les objectifs assignés aux politiques de santé animale. Ces politiques associent des objectifs nombreux et potentiellement contradictoires de santé publique, d'économie agricole, de commerce international, de bien-être animal, et plus récemment, d'en faire une composante du triptyque One Health. Ce dernier objectif est lié à la mobilisation de la communauté internationale (FAO, OMS et OIE [Organisation mondiale de la santé animale]) pour la santé globale. La santé animale devient simultanément un enjeu de biosécurité et un bien public mondial. Ce recadrage permet de mobiliser la communauté internationale sur le registre de la menace et de l'intérêt général. L'exemple de la grippe aviaire au Vietnam montre la nécessité de veiller à ce que ce recadrage ne marginalise pas les enjeux et les apprentissages locaux. Plus généralement, la santé animale est un objet politique qu'il faut dénaturaliser. Les sciences sociales permettent de comprendre les intérêts, les valeurs en concurrence dans le concept de santé animale et de nourrir le débat sur ce que nous voulons en faire pour construire un monde plus sûr mais aussi plus solidaire entre États ainsi qu'entre humains et animaux.
\end{abstract}

Mots-clés : globalisation / organisation internationale / maladie émergente / risque / Vietnam

\begin{abstract}
Animal health governance: between biosecurity and a global public good. The history, definition and objectives of animal health policies are reviewed in this article. These policies combine numerous potentially contradictory objectives in public health, agricultural economics, international trade and animal welfare, while recently also being a component of the One Health triptych. This latter objective emerged as a consequence of the drive of the international community (FAO, WHO and OIE [World Organisation for Animal Health]) to achieve global health. Animal health is becoming both a biosecurity issue and a global public good. This reframing helps mobilize the international community according to the scale of the threat and the general interest. The case of the avian influenza in Vietnam showcases the need to ensure that this reframing does not marginalize local issues and knowledge. Animal health is a political target that needs to be denaturalized. Social sciences can highlight the competing interests and values at stake in the animal health concept, while nurturing the debate on how it could help build a safer world and enhance solidarity between States, as well as between humans and animals.
\end{abstract}

Keywords: emerging disease / globalization / international organization / risk / Vietnam

\footnotetext{
* Auteur correspondant : muriel.figuie@cirad.fr
} 
L'objectif de cet article est d'analyser les politiques publiques de santé animale et leurs récentes évolutions dans un contexte de globalisation. Depuis leurs débuts en Europe au $\mathrm{XVIII}^{\mathrm{e}}$ siècle, ces politiques ont connu d'importantes transformations qui témoignent de la place croissante, mais aussi ambiguë, qu'elles occupent dans le registre de l'action publique, associant des objectifs potentiellement contradictoires de santé publique, d'économie agricole, de commerce international, de bien-être animal. Plus récemment, ces politiques sont considérées comme la composante d'un ensemble associant santé humaine, animale et environnementale à travers le concept «Une seule Santé/One Health $(\mathrm{OH}) »$. L'Europe, et en particulier la France et la Grande-Bretagne, a joué un rôle central dans l'histoire de ces politiques (Woods et Bresalier, 2014; Bonnaud et Fortané, 2018). Les organisations internationales [principalement la FAO (Organisation des Nations unies pour l'alimentation et l'agriculture), l'OIE (Organisation mondiale de la santé animale) ${ }^{1}$ et l'OMS (Organisation mondiale de la santé)] ont participé à leur internationalisation (Figuié, 2014; Teissier, 2007 ; Vagneron, 2015). Les crises sanitaires comme celle de la grippe aviaire au Vietnam en 2004 ont fortement contribué à promouvoir une gouvernance zoosanitaire mondiale reposant sur les concepts de biosécurité et de bien public mondial. Ce sont ces transformations que nous examinons et analysons ici.

L'apport des sciences sociales à l'étude des politiques de santé animale peut se résumer à trois domaines de recherche. Le premier privilégie une approche historique et montre que le développement des politiques de santé animale en France, en Grande-Bretagne et dans le contexte colonial (Swabe, 1999; Berdah, 2010; Brown et Gilfoyle, 2010; Woods, 2004; Barroux, 2011) est fortement lié à la profession vétérinaire (Swabe, 1999; Berdah, 2010 ; Brown et Gilfoyle, 2010; Woods, 2004 ; Barroux, 2011). Les auteurs associent l'émergence de la santé animale comme objet de l'action publique à la création en France de la première école vétérinaire et d'un corps vétérinaire par décret royal en 1761. Ce corps va s'opposer aux savoirs empiriques. Bénéficiant du soutien de l'État, il concurrencera les maréchauxferrants, barbiers et guérisseurs dans les soins aux animaux de rente (Woods et Bresalier, 2014). Le contenu des politiques et des enjeux de santé animale est à la fois le produit des missions négociées entre l'État et le corps vétérinaire (Hubscher, 1999) et de la place de l'animal

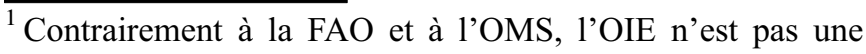
organisation des Nations unies. C'est une organisation intergouvernementale créée en 1924 qui rassemble aujourd'hui 182 États membres (son sigle, OIE, est héritée de son ancien nom: Organisation internationale des épizooties).
}

(Swabe, 1999) et de l'élevage (Woods, 2004) dans la société. Dans le contexte européen du XVIII siècle (conflits entre États, développement du commerce de longue distance, urbanisation), l'enjeu est de préserver un potentiel de guerre (les chevaux), un potentiel économique (le commerce du bétail menacé par de grandes épizooties ${ }^{2}$ ) et de sécuriser l'approvisionnement alimentaire des villes. Au cours du $\mathrm{XIX}^{\mathrm{e}}$ siècle, la profession se médicalise en se rapprochant de la communauté pasteurienne pour prendre en charge les maladies animales infectieuses transmissibles aux humains (les zoonoses) et la sécurité sanitaire des aliments, jusqu'à obtenir des autorités publiques le monopole de la gestion des maladies contagieuses en élevage et de l'inspection des viandes (Hubscher, 1999; Stanziani, 2005).

Un deuxième domaine de recherche a été encouragé par les crises sanitaires (vache folle, fièvre aphteuse...) du tournant du siècle dernier. Ces crises offrent l'opportunité d'élargir à de nouveaux domaines (l'agriculture et l'alimentation) les travaux antérieurs (Lagadec, 1981; Godard et al., 2002; Gilbert, 2003) conduits sur les risques associés aux innovations (nucléaire, amiante...) et qui confrontent différentes perceptions des risques (expertes et profanes), interpellent le fonctionnement des organisations ainsi que les relations entre sciences du vivant, techniques et sociétés. S'ils n'abordent pas directement la santé animale en tant qu'objet politique, ces travaux mettent en lumière les évolutions de la profession (Bonnaud et Fortané, 2021a ; 2021b) et le rôle ambigu des vétérinaires, entre conseillers agricoles et garants de la santé publique, entre protecteurs de l'intérêt des éleveurs et de la santé des consommateurs (Bonnaud et Fortané, 2016 ; 2018). Ils permettent également dans le prolongement de travaux initiés par Wynne (1996) de mettre en évidence les différentes ontologies des maladies animales (Law et Mol, 2011; Enticott, 2012).

Un troisième corpus de travaux plus récents porte sur l'émergence du concept «Une seule Santé/One Health » $(\mathrm{OH})$ et sa mise en œuvre. Le concept invite d'une part à rapprocher santé animale et santé humaine dans le domaine scientifique comme politique (FAO et al., 2008). L'idée n'est pas nouvelle (Woods et Bresalier, 2014; Zinsstag et al., 2011) mais prend de l'ampleur depuis la fin du $\mathrm{XX}^{\mathrm{e}}$ siècle avec la multiplication de nouvelles maladies infectieuses affectant les humains (Sida, grippe aviaire, maladie à virus Ébola, maladies à Coronavirus...) et dont la majorité est d'origine animale (Jones et al., 2008). D'autre part, à travers les liens établis entre crise environnementale et multiplication des maladies émergentes (Morand, 2016), c'est également la

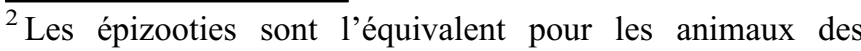
épidémies chez les humains.
} 
santé de l'environnement que les promoteurs du concept $\mathrm{OH}$ invitent à prendre en compte. Enfin, parce que ces maladies ne connaissent pas de frontière, elles contribuent à légitimer et à renforcer le rôle des organisations internationales (OI) qui en sont d'importants promoteurs [voir, par exemple, FAO et al. (2008)]. Les travaux de sciences sociales portant sur le concept $\mathrm{OH}$ interrogent les frontières et les relations entre humains et non-humains (Keck, 2012; Porter, 2012), mais aussi sa capacité de mobilisation (Michalon, 2019), les frontières entre les domaines de l'action publique et les concurrences juridictionnelles que sa mise en œuvre contribue à réorganiser (Jerolmack, 2013; Gardon et al., 2019) en particulier au niveau international (Chien, 2013; Figuié, 2014).

Notre contribution relève de ce dernier corpus. La plupart des travaux analysent ces évolutions comme un appel à l'interdisciplinarité, à la multisectorialité et à la coopération internationale, en privilégiant un point de vue de santé publique et sans entrer dans le détail des reconfigurations de la santé animale que cela suppose. Nous montrons que ces évolutions contribuent à élargir le cadrage de la santé animale pour en faire un enjeu de biosécurité ainsi qu'un nouveau bien public mondial.

L'objectif de la biosécurité est de protéger le vivant par le contrôle des flux de différentes formes de vies (humains, animaux, bactéries, virus, gènes...), flux associés à des risques d'invasions biologiques, contaminations, sauts d'espèces, bioterrorisme... Le cadrage biosécuritaire repose sur le registre de la menace et de la peur. Dans le domaine de la santé animale, les études portent principalement sur les dispositifs de contrôle et de surveillance à l'échelle des élevages [voir les contributions rassemblées par (Fortané et Keck, 2015) ou (Bingham et al., 2008)]. Mais les effets de ce recadrage biosécuritaire sur les politiques internationales ont été peu étudiés dans le secteur de la santé animale contrairement au secteur de la santé publique (Lakoff et Collier, 2008).

Les biens publics mondiaux (BPM) sont définis comme «l'ensemble des biens accessibles à tous les États qui n'ont pas nécessairement un intérêt individuel à les produire » (Kindleberger, 1988). Le concept met en avant la défaillance des marchés (cas des biens publics), mais également des États (cas des BPM) pour leur production et leur prise en charge (Gabas et Hugon, 2001) et la nécessité d'une gouvernance mondiale de ces biens. Le concept a d'abord été appliqué au système monétaire et au commerce international (Kindleberger, 1988) avant d'être étendu à l'environnement et à la santé (Brown et al., 2006). Le cadrage en termes de BPM permet un changement d'échelle et la mobilisation d'un spectre plus large d'acteurs, mais surtout il introduit un nouveau régime de régulation de la santé. Contrairement à la santé animale, son usage en santé humaine a fait l'objet de travaux en sciences sociales, notamment pour en analyser l'ambiguïté du fait de la dimension également marchande de la santé (Boidin, 2014; Kerouedan, 2013 ; Smith et al., 2004).

Nous montrons dans cet article (1) la polysémie du terme de santé animale et ce qu'elle révèle de la multiplicité et des ambiguiités des enjeux liés aux politiques de santé animale. Puis nous analysons les transformations récentes de ces politiques : (2) leur cadrage en termes de biosécurité et (3) en termes de bien public mondial. Enfin, nous illustrons (4) les effets de ces recadrages à partir du cas de la gestion de la grippe aviaire au Vietnam.

Pour procéder à cette analyse, nous nous appuyons sur plusieurs matériaux: (1) l'abondante littérature grise produite par les institutions faisant autorité en santé animale (ministère de l'Agriculture, Académie vétérinaire en France, Union européenne, FAO, OIE, OMS) ; (2) une vingtaine d'entretiens conduits auprès des acteurs de ces politiques, principalement dans le contexte de gestion de la grippe aviaire (H5N1) au niveau international (FAO, OIE et OMS) et national [Vietnam] (Figuié, 2014 ;2013); et (3) une quarantaine d'entretiens auprès d'éleveurs du district de Phu Xuyen (Figuié et Desvaux, 2015), et d'acteurs institutionnels et professionnels de la filière avicole au Vietnam (Figuié et al., 2013).

\section{Une définition institutionnelle de la santé animale à dominante utilitariste}

Il est difficile de trouver une définition de la santé animale elle-même. Les définitions se réfèrent le plus souvent à la santé publique vétérinaire; la santé animale n'est définie qu'indirectement, par ses objectifs. Diverses institutions se réfèrent ainsi à travers l'énoncé de leur mandat à la santé publique vétérinaire (ministère de l'Agriculture et Académie vétérinaire en France; OIE, FAO...) et fixent les objectifs de la santé animale ou du contrôle des maladies animales (Union européenne). Dans tous les cas, c'est l'animal d'élevage qui est de façon plus ou moins implicite au cœur des énoncés, la responsabilité des ministères en charge de l'agriculture qui est engagée, et la science vétérinaire qui est présentée comme la détentrice légitime de l'expertise sur le sujet.

Les principales OI concernées (FAO, OIE, OMS) ont convenu de définir la santé publique vétérinaire comme «la somme de toutes les contributions au bien-être physique, mental et social de l'Homme à travers la connaissance et l'application de la science vétérinaire ${ }^{3} »$. Et la Commission des communautés euro-

\footnotetext{
${ }^{3}$ Définition adoptée par la FAO, l'OIE et l'OMS à la conférence de Teramo en 1999, www.fao.org/3/y4962t/y4962t01.htm.
} 
péennes (CCE, 2007) résume ainsi les objectifs de sa stratégie pour la santé animale:

- «Garantir un niveau élevé de protection de la santé publique et de la sécurité alimentaire en réduisant l'incidence des risques biologiques et chimiques sur l'être humain au minimum;

- Promouvoir la santé animale en prévenant/réduisant l'incidence des maladies des animaux et, par là même, soutenir l'élevage et l'économie rurale;

- Améliorer la croissance économique/la cohésion/la compétitivité en assurant la libre circulation des marchandises et les nécessaires mouvements des animaux;

- Promouvoir des modes d'élevage et une politique du bien-être animal qui préviennent les menaces liées à la santé animale et minimisent les retombées sur l'environnement afin de soutenir la stratégie de développement durable de l'UE.»

Ces trois premières dimensions (santé humaine, performances de production, commerce) font de la santé animale un levier du développement et une composante majeure de l'intervention de la FAO car «les maladies qui touchent le bétail peuvent avoir des effets dévastateurs sur la productivité et la production animales, le commerce d'animaux sur pied, de viande et autres produits d'origine animale, sur la santé humaine et, partant, sur l'ensemble du processus de développement économique ${ }^{4} \gg$.

Ces éléments de cadrage appellent plusieurs remarques. Premièrement, la santé animale est abordée comme un instrument au service des humains et les maladies animales comme des risques pour ceux-ci. Elle est au service de divers objectifs : santé publique, performance technico-économique, commerce. Même le bien-être animal y est abordé dans une perspective de développement (certes durable). Cette instrumentalisation est encore plus évidente si on compare la santé animale à la santé humaine telle que définie par l'OMS : la santé est « un état de complet bien-être physique, mental et social, et ne consiste pas seulement en une absence de maladie ou d'infirmité "». C'est «l'Homme» et non pas l'animal qui se trouve au cœur des politiques de santé animale.

La deuxième remarque porte sur l'ampleur des objectifs assignés à la santé animale. De nombreux domaines d'expertise, au-delà de la seule expertise vétérinaire, et secteurs d'intervention, au-delà du seul secteur agricole, peuvent s'en revendiquer les «propriétaires légitimes », ouvrant la porte à de potentielles concurrences. Ainsi dans le passé, l'expertise vétérinaire

\footnotetext{
${ }^{4}$ www.fao.org/ag/againfo/themes/fr/animal health.html.

${ }^{5}$ Source : préambule à la Constitution de l'ŌMS, adoptée par la Conférence internationale sur la Santé, New York, 19-22 juin 1946. La définition n'a pas été modifiée depuis 1946.
}

s'est trouvée en diverses occasions en compétition avec celle de la médecine lorsqu'il s'agit de maladies zoonotiques (maladies transmissibles des animaux aux humains) ${ }^{6}$; avec celle des zootechniciens (Landais, 1990) en particulier lorsqu'il s'agit de maladies de production [liées au mode d'élevage, Joshi et Herdt (2006) ; Bonnaud et Fortané (2021b)]; avec celle des écologues (Jerolmack, 2013) lorsqu'il s'agit de maladies transmises par la faune sauvage, etc.

Le troisième constat est que la compatibilité des objectifs assignés aux politiques de santé animale est rarement questionnée. Pourtant, plusieurs exemples attestent de contradictions, voire de conflits d'intérêts : la gestion de la vache folle a montré la difficulté pour le ministère de l'Agriculture français de défendre simultanément la santé des consommateurs et les intérêts économiques de la filière bovine (Barbier, 2002); de même, les abattages massifs d'animaux (y compris d'animaux sains) lors des épizooties passées de fièvre aphteuse en Angleterre visent une reprise rapide du commerce international et sont peu cohérents avec un objectif de bien-être animal.

Enfin, l'émergence du paradigme Une seule Santé/ One Health $(\mathrm{OH})$ invite à décloisonner dans la recherche et dans l'intervention les approches de santés humaine, animale et environnementale. Cependant, dans la pratique, sa portée est réduite: le paradigme $\mathrm{OH}$ reste anthropocentré et focalisé sur les zoonoses. C'est seulement à travers l'émergence de considérations en faveur du bien-être animal (limitées comme souligné plus haut) ou à travers l'animal de compagnie qu'une convergence dans les définitions apparaît entre santés humaine et animale. À noter également une inflexion notable récente au sein de l'Académie vétérinaire française : la définition de la santé publique vétérinaire adoptée par la FAO, l'OIE et l'OMS citée plus haut est directement empruntée à la définition de l'Académie vétérinaire française de 1997. Cette dernière a été révisée (et élargie) pour intégrer les évolutions récentes apportées par le concept «Une seule santé »?

\footnotetext{
${ }^{6}$ Sont inclues parfois dans la définition des zoonoses les maladies transmissibles des humains aux animaux, mais beaucoup plus rarement, témoignant là encore de la vision anthropocentrique de la santé animale.

7 «La santé publique vétérinaire est l'ensemble des actions collectives, principalement régaliennes, en rapport avec les animaux sauvages ou domestiques, leurs services et leurs productions entrant notamment dans la chaîne alimentaire, qui visent à préserver les santés humaine et animale - y compris l'état de bien-être - et la santé des écosystèmes. Elle contribue ainsi au développement durable et à la mise en œuvre du concept «Une seule santé».» (Académie vétérinaire de France, juin 2017), https://academie-veterinaire-defrance. org/actualites/communique-de-presse-2021-08-lacademieveterinaire-de-france-revisite-la-definition-de-la-sante-publi que-veterinaire.
} 
Ces considérations en matière de bien-être restent cependant négligeables face à l'importance croissante que prennent les enjeux de biosécurité et l'instrumentalisation de l'animal qu'ils contribuent à renforcer, comme nous le montrons plus bas.

L'examen des pratiques discursives des organisations en charge de la santé animale montre que celle-ci est loin d'être un concept biotechnique clairement circonscrit tant les objectifs sont multiples (santé humaine, économie, commerce, développement, sécurité, bienêtre animal). Le constat est celui d'un manque de clarté entre les différents termes utilisés. Par exemple, l'OIE se définit comme "l'organisation mondiale de la santé animale ${ }^{8}$ » ayant pour mandat « la santé animale, la santé publique vétérinaire et le bien-être animal », et considère que « la santé animale est une composante fondamentale du bien-être des animaux ${ }^{9} »$. Ces ambiguïtés reflètent les frontières mouvantes des politiques de santé animale et la place ambivalente qu'elles occupent dans le registre de l'action publique. C'est autour des enjeux de biosécurité et le concept de bien public mondial que ces frontières évoluent aujourd'hui comme nous le montrons dans la suite de l'article.

\section{Les maladies animales, risques et biosécurité}

Comme dans d'autres domaines de l'action publique, les problèmes de santé animale sont gérés à travers des dispositifs standardisés d'analyse des risques. Cependant, depuis quelques décennies, la pertinence de cette approche est remise en cause par l'émergence de nouveaux enjeux sanitaires impliquant l'animal.

\section{Risques et menaces}

L'analyse des risques, d'abord conceptualisée par les Académies des sciences aux États-Unis (Demortain, 2019), est devenue un standard international. La gestion des problèmes en tant que risques est une modalité d'action publique qui a émergé avec le développement scientifique au siècle des Lumières; et celle des problèmes de santé animale (qui fait l'objet de cet article) prend forme avec l'institutionnalisation d'un art vétérinaire (voir plus haut). C'est une gestion dite moderne des dangers qui défend sa légitimité en référence à la science et en opposition aux démarches empiriques ou magico-religieuses (Swabe, 1999). Aujourd'hui des outils standardisés de maîtrise des

\footnotetext{
${ }^{8}$ www.oie.int/fr.

${ }^{9}$ Recommandations issues de la quatrième conférence mondiale de l'OIE sur le bien-être animal, Guadalajara (Mexique), 2016.
}

risques sont partagés internationalement; ils comprennent des étapes d'analyse (identification des dangers), d'évaluation (calcul des probabilités), de gestion (définition des mesures de contrôle) et de communication (pour connaître l'acceptabilité des risques et faciliter la mise en œuvre des mesures de contrôle).

La succession de crises que l'on connaît depuis ces mêmes années 1980 (Three Miles Island, Bophal, Tchernobyl, Sida, Vache folle...) a conduit à mettre à l'agenda de «nouveaux risques» (Godard et al., 2002) dont la gestion pose de nouveaux défis du fait de leur puissance catastrophique (ces risques sont potentiellement de grande ampleur), leurs nombreuses causes et implications (risques complexes et systémiques), leur possible extension géographique (risques globaux) et leurs effets sur le long terme (ils peuvent toucher les générations futures et avoir des effets irréversibles). Ces nouveaux risques se «soustraient au travail de réduction d'incertitude introduit par la notion même de risque » (Gilbert, 2002) et divers auteurs suggèrent de parler alors de «menaces » [c'est le choix de cet article] (Bourg et al., 2013; Godard et al., 2002) ou de risques de deuxième modernité (Beck, 2001). Face aux menaces, les outils classiques de gestion et de prévention (reposant sur une analyse statistique d'événements passés liés à des dangers bien identifiés) sont inopérants pour appuyer la décision publique. On passe ainsi d'une gestion de risques avérés (dont les causes et les probabilités sont connues) à celle de menaces (incertaines, complexes, imprévisibles). Ce recadrage se traduit par la contrainte d'agir en situation d'incertitude (Callon et al., 2001; Godard et al., 2002). Dans le domaine du vivant et des risques sanitaires en particulier, cette évolution a contribué à l'adoption du concept de biosécurité.

\section{Maladies émergentes et biosécurité}

Le passage du contrôle du risque sanitaire à la biosécurité est lié à l'ambition de ne plus limiter l'action publique à la prévention classique des risques avérés, pour $\mathrm{y}$ inclure la préparation aux menaces, aux catastrophes à venir et à la gestion de risques incalculables. Dans le domaine de la santé, le concept de maladies émergentes forgé dans les années 1990 a donné une base scientifique à cette ambition (King, 2004), ambition qui s'est affirmée avec l'apparition de nouveaux agents infectieux (VIH, prion, virus grippaux, virus Ébola, coronavirus...) et leur diffusion à large échelle.

Les politiques de biosécurité sont un prolongement des biopolitiques (Foucault, 2004): ces dernières reposent sur un contrôle des risques associés au vivant, grâce à des dispositifs de production de statistiques, de surveillance et de contrôle des conduites individuelles. Les politiques de biosécurité intègrent la menace, 
l'incertitude et le risque de catastrophe. Fortané et Keck rappellent qu'en sciences sociales, le concept de biosécurité est apparu pour décrire une nouvelle rationalité du risque dans la gestion du vivant au croisement de logiques sanitaires et militaires:

«Alors que les formes antérieures de biopolitique reposaient sur des modèles statistiques permettant de prévoir et de mesurer les risques, la biosécurité implique de se préparer à une catastrophe dont la probabilité est incalculable et dont l'arrivée est perçue comme imminente (Lakoff et Collier, 2008). L'émergence d'un nouveau pathogène est ainsi vue comme un événement auquel les autorités sanitaires doivent se préparer en imaginant ses conséquences encore incertaines pour la population humaine» (Fortané et Keck, 2015, p. 125).

Du fait de l'origine animale de la plupart des maladies infectieuses, la maîtrise des zoonoses devient une composante majeure des politiques de santé animale et de santé publique. Par ailleurs, avec les maladies émergentes prend fin l'idée qu'il existe un nombre fini de maladies que les progrès scientifiques permettent d'éliminer une à une. Le modèle pastorien de l'éradication est mis à mal. Les maladies ressemblent aujourd'hui à un ensemble toujours renouvelable d'entités instables et innombrables: les virus (comme ceux de la grippe notamment) mutent de façon continue et aléatoire, l'élimination d'une maladie peut laisser le champ libre à une autre (selon certains spécialistes, l'élimination de la peste bovine aurait favorisé la peste des petits ruminants) et les outils de maîtrise tels les antibiotiques créent des résistances et de nouveaux pathogènes. Aucun progrès n'est acquis, la vigilance doit être permanente.

Pour la FAO, la biosécurité recouvre un ensemble de mesures visant à limiter la diffusion et l'incidence de maladies (animales ou végétales) présentes ou à venir, et en particulier à éviter que les agents pathogènes ne réalisent des "sauts d'espèces» et ne passent des barrières géographiques. Ces mesures sont essentiellement des mesures de surveillance pour la production d'informations sanitaires permettant de déclencher l'alarme, et de préparation à de potentielles catastrophes selon la formule commune «not if, but when». Elles permettent de repérer des animaux porteurs d'agents pathogènes qui sans être eux-mêmes malades (les chauves-souris sont ainsi les réservoirs et porteurs sains de nombreux pathogènes pour les humains) représentent une menace potentielle : le champ de la santé animale est ainsi beaucoup plus vaste que celui des seuls animaux malades et l'animal sauvage y occupe une place croissante. L'étude de l'origine des crises sanitaires récentes montre que les animaux (rats, chauves-souris, pangolins, civettes...) peuvent être à l'origine de menaces susceptibles de bouleverser en profondeur la vie économique, sociale et politique à l'échelle planétaire. La logique biosécuritaire progresse également avec l'idée selon laquelle, comme les maladies humaines, les maladies animales peuvent devenir des armes biologiques: en 2016, l'OIE a élaboré une «Stratégie pour la réduction des menaces biologiques. Renforcer la sécurité biologique mondiale» fournissant une liste des agents pathogènes d'origine animale pouvant servir d'armes ${ }^{10}$.

Le registre de la peur, propre au domaine de la menace et de la biosécurité, se substitue à celui de la confiance mis en avant dans le monde des risques probabilisés et de la prévention. Plusieurs auteurs (Dean, 1999; Ewald, 1986) proches des analyses en termes de biopolitiques voient dans la gestion des menaces et des risques incalculables l'occasion d'un contrôle croissant des États sur les individus par une surveillance accrue (Taylor-Gooby et Zinn, 2006; Ewald, 1986; Buton, 2008). D'autres auteurs dont Beck (2009), plus optimistes, voient dans les risques globaux l'opportunité d'un «tournant démocratique» et "qu'émerge une culture de responsabilité qui transcende les frontières et les conflits ${ }^{11}$ »; la capacité de prendre en charge des risques globaux témoignerait ainsi d'un processus de civilisation (Elias, 1973).

Loin de s'opposer, nous montrons dans la partie suivante, avec la santé animale, que ces tendances sont complémentaires. À la faveur des zoonoses émergentes, la santé animale évolue pour englober des enjeux de biosécurité. Ces enjeux sont globaux. Le cadrage en termes de BPM permet non seulement un changement d'échelle, mais il élargit aussi les responsabilités des États et les mobilise par la référence à l'intérêt commun et à une morale de responsabilité. La peur (associée à la biosécurité) oriente l'attention vers les biens à protéger, l'intérêt commun (porté par le concept de BPM) permet l'action collective pour la protection de ces biens. C'est ce que nous détaillons dans la partie suivante.

\section{La santé animale : coopération et bien public mondial}

Les objectifs assignés à la santé animale (sécurisation de la production et du commerce agricoles, de la qualité sanitaire des aliments et santé publique) en font un bien public et un objet des politiques publiques nationales. Ces politiques font également l'objet d'accords de coopérations entre les États visant à soutenir le commerce international (à travers l'élaboration et le contrôle de normes sanitaires internationales) et l'aide au développement (à travers des programmes bilatéraux ou

\footnotetext{
${ }^{10}$ Voir le rapport de 1'OIE publié en 2016: www.oie.int/ fileadmin/Home/fr/Our_scientific_expertise/docs/pdf/F_Biolo gical_Threat-Reduction_Strategy_jan2012.pdf.

${ }^{11} \mathrm{Ma}$ - traduction de 'a culture of responsibility that transcends borders and conflicts'.
} 
multilatéraux de soutien aux problèmes zoosanitaires locaux). Plus récemment, en tant que composante du triptyque One Health, la santé animale comme avant elle la santé humaine (et dans une moindre mesure la santé de l'environnement), se trouve engagée dans un processus de globalisation associé à l'acquisition du statut de bien public mondial. Ce sont ces étapes que nous retraçons ici, en soulignant les changements majeurs qui les caractérisent.

\section{Politiques publiques, coopération internationale et globalisation}

En Europe, la santé des animaux d'élevage a été longtemps considérée par les autorités publiques comme une affaire privée relevant principalement de la décision des propriétaires d'animaux, même si des règles collectives de gestion existaient probablement. L'action publique portait essentiellement sur la qualité sanitaire des viandes commercialisées (Stanziani, 2005). C'est dans les années 1840 (Teissier, 2007), avec la promulgation des premières lois, que la santé des animaux d'élevage est devenue un objet public. Cette prise en charge était étroitement associée à la volonté des États d'accompagner la modernisation du secteur agricole, en le protégeant notamment des menaces infectieuses, en particulier des grandes épizooties de peste bovine et fièvre aphteuse (Woods, 2004).

Les politiques de santé animale s'inscrivirent également dans l'objectif de sécurisation des échanges internationaux en Europe et avec les colonies (Woods, 2004). Ainsi, depuis le $\mathrm{XIX}^{\mathrm{e}}$ siècle, les politiques publiques nationales de gestion de la santé animale sont liées aux politiques de coopération internationale et ceci de façon plus concrète depuis la création de l'OIE en 1924. Cette coopération vise à permettre aux États de protéger leur territoire des menaces infectieuses extérieures sans entraver le commerce international, en s'entendant sur les garanties sanitaires exigibles entre États. Le but est, d'une part, de protéger les pays importateurs du risque d'introduction de maladies animales sur leur territoire via le commerce (risques pour leur cheptel et pour la santé publique); il est, d'autre part, de protéger les pays exportateurs des tentatives éventuelles de la part des pays importateurs d'invoquer abusivement des raisons sanitaires à des fins de protectionnisme économique (Teissier, 2007). Ce dispositif s'est consolidé par la suite avec la création de l'Organisation mondiale du commerce (OMC) qui, en 1998, a fait des normes élaborées par l'OIE ses normes de références.

La santé animale est également une composante des politiques internationales d'aide au développement (voir plus haut). L'objectif est de soutenir les services vétérinaires des pays sous régime d'aide dans leur lutte contre les maladies animales qui impactent localement la santé des populations ou la productivité des élevages (trypanosomiases, maladies à tiques...). Ces politiques s'appuient sur des dispositifs multilatéraux dans lesquels la FAO, créée en 1945, joue un rôle majeur, s'appuyant notamment sur l'OIE grâce à un accord passé entre les deux organisations dès 1952 . Les interventions relevant de ces politiques sont centrées sur des enjeux locaux, dans une perspective de solidarité internationale (même si les intérêts des pays donateurs peuvent parfois interférer dans la hiérarchisation des enjeux et le choix des dispositifs de développement).

Jusqu'à la fin du $\mathrm{XX}^{\mathrm{e}}$ siècle, les coopérations entre États s'inscrivaient dans des politiques internationales plutôt que globales, pour reprendre la distinction opérée en santé humaine par divers auteurs (Kerouedan, 2013; Fidler, 2003; Brown et al., 2006; Boidin, 2014). Le concept de santé publique internationale a précédé celui de santé publique globale au sein des OI: la santé internationale est axée sur les problèmes des pays du Sud, sur les maladies qui y pèsent le plus lourdement sur les populations; la santé globale, qui s'est développée à la fin du $\mathrm{XX}^{\mathrm{e}}$ siècle, met au contraire en avant les problèmes globaux, c'est-à-dire ceux qui concernent à la fois les pays du Nord et les pays du Sud. Divers auteurs soulignent leurs préoccupations face à la montée en puissance de ce concept dans les instances de gouvernance sanitaire mondiale: pression croissante sur les pays pauvres pour les amener à prendre en charge des catastrophes potentielles quand ils n'ont déjà pas les ressources nécessaires pour faire face aux maladies infectieuses «classiques» comme la méningite ou la malaria (Calain, 2007) qui les affectent; mise au second plan par les OI des priorités sanitaires nationales des pays du Sud au profit des problèmes globaux (Kerouedan, 2013).

Cette transition de la santé internationale vers la santé globale s'est faite à la faveur de la multiplication des maladies infectieuses émergentes et de leur recadrage en tant qu'enjeux de sécurité globale. La santé animale s'y trouve associée du fait du caractère zoonotique fréquent de ces maladies et de la montée en puissance du paradigme $\mathrm{OH}$. C'est ce processus de globalisation (en opposition à celui d'internationalisation) et en lien avec les enjeux de biosécurité que nous examinons ci-dessous dans le domaine de la santé animale.

\section{Santé et sécurité globales}

Depuis le début du $\mathrm{XXI}^{\mathrm{e}}$ siècle, plusieurs éléments discursifs témoignent d'un cadrage croissant de la santé comme enjeu de sécurité globale. Ainsi, en 2003, dans le contexte de l'épidémie mondiale de Sras (syndrome 
respiratoire aigu sévère), la CIA (Central Intelligence Agency) organisait une réunion d'experts en santé publique, économie et communication. Évoquant le Sras ${ }^{12}$ comme «la première pandémie du $\mathrm{XXI}^{\mathrm{e}}$ siècle», les participants concluaient: «Si le Sras avait été un peu plus contagieux, nous n'aurions pas pu le contenir... Le Sras a été un avertissement qui doit faire réfléchir sur les graves conséquences dans le monde qui peuvent se produire à tous les niveaux, sanitaire, économique, et politique lorsque des épidémies imprévues surviennent dans un monde interconnecté et accéléré » (CIA, 2003, ii et iv) ${ }^{13}$. Cette menace globale exige une réponse globale : «Aujourd'hui plus que jamais la propagation internationale des maladies ou autres risques menace la santé et l'économie et la sécurité. Aucun pays ne peut protéger seul ses citoyens contre ces menaces ${ }^{14} \gg$ selon le rapport annuel de l'OMS sous-titré en 2007 A safer future: global public health security (OMS, 2007).

Parce que la plupart des maladies infectieuses émergentes ont une origine animale, le mouvement touche également la santé animale. L'animal se trouve ainsi étroitement associé à la menace pandémique ${ }^{15}$. Quelques années après l'OMS, la FAO (2013) fait de la sécurité sanitaire globale (global health security) le thème central de son rapport annuel. Et les concepts d'agrocrime et d'agroterrorisme sont à la base de nouvelles collaborations entre l'OIE, la FAO et Interpol (l'Organisation internationale de police criminelle) ${ }^{16}$. Ces éléments confirment le cadrage biosécuritaire de la santé animale mais aussi sa globalisation.

La globalisation de la santé animale se traduit par une évolution de sa gouvernance internationale. La santé globale est plus que la somme de la santé de chaque pays, de même que $\mathrm{OH}$ est plus que la somme de la santé humaine, animale et environnementale, et que le tout est plus que la somme des parties. La santé globale instaure un régime de responsabilité nouveau: les États sont responsables du contrôle de l'entrée et de la circulation

\footnotetext{
${ }^{12}$ Le Sras est arrivé depuis la Chine au Vietnam en 2003. Il s'est ensuite propagé dans 29 pays en quelques mois faisant près de 900 victimes.

${ }^{13}$ Ma traduction de 'Had SARS been even moderately more contagious, it probably could not have been contained' and 'SARS has served as a sobering warning about the serious worldwide consequences that can occur at every level, public health, economic, and political when unanticipated epidemics arise in a highly connected, fast-paced world'.

${ }^{14} \mathrm{Ma}$ traduction de 'Today more than ever the international spread of diseases or other risks threatens health, economies and security. No country can "go it alone" in protecting its citizens from the threats'.

${ }^{15}$ Une pandémie est une épidémie d'échelle mondiale.

${ }^{16}$ https://oiebulletin.com/?panorama=02-1-2-2020-2_oie-faointerpol.
}

d'agents pathogènes sur leur propre territoire mais, et c'est là que réside la nouveauté, ils ont aussi la responsabilité d'éviter la «sortie» d'agents pathogènes (ou même potentiellement pathogènes) d'origine humaine ou animale représentant une menace pour la santé dans les autres pays et plus précisément pour le maintien de flux (personnes, marchandises...) à la base du fonctionnement d'un monde globalisé. C'est ainsi que le directeur de l'OIE de l'époque déclarait: «De fait, un seul pays qui serait aujourd'hui dans l'incapacité de lutter contre les foyers de maladies animales pourrait mettre en danger la planète entière » (Vallat, 2007). Cette interdépendance nouvelle que créent les enjeux de biosécurité à l'échelle globale, ainsi que la défaillance de certains États, permet de justifier l'intervention de la communauté internationale dans ce qui était auparavant considéré comme relevant d'affaires purement nationales. En 2006 le président de la mission d'information sur la grippe aviaire (France), évoquant un changement de paradigme, plaidait pour un droit d'ingérence sanitaire dans le domaine de la santé humaine et animale ${ }^{17}$ : «Aujourd'hui, nous avons atteint un degré d'exigence supplémentaire (envers les gouvernements)... Les États développés ont naturellement le devoir d'aider les pays qui ne disposent pas des moyens d'une politique de santé, mais également celui d'exiger une transparence complète de l'information sur l'évolution de l'alerte et la mise en œuvre d'une véritable gouvernance sanitaire ${ }^{18}$ ».

L'élargissement de la responsabilité promu à travers la santé globale est loin d'être accepté par tous les États soucieux du maintien de leur souveraineté (Figuié, 2013). Pour mobiliser autour de cette responsabilité nouvelle, les OI impliquées dans la santé animale s'appuient sur le concept de bien public mondial et la référence à l'intérêt général.

\section{La santé animale, bien public mondial}

Dans un document de 2008 publié suite à l'épizootie de grippe aviaire (H5N1) et établissant une stratégie commune pour réduire les risques de maladies infectieuses humaines et animales, les principales OI concernées (FAO, OIE, OMS, Banque mondiale...) écrivent: "La prévention de l'émergence et de la propagation transfrontalière de maladies infectieuses humaines et animales est un bien public mondial... Les systèmes de surveillance sur lesquels repose la préven-

\footnotetext{
${ }^{17}$ J.-M. Le Guen, président de la mission d'information parlementaire sur la grippe aviaire, le 11 janvier 2006 www. assemblee-nationale.fr/12/miga/05-06/c0506023.asp\#TopOf Page.

${ }^{18}$ www.lemonde.fr/l-epizootie-de-grippe-aviaire/article/2006/ $01 / 16 / m-1 e-g u e n-p o u r-u n-d r o i t-d-i n g e r e n c e-s a n i$ taire_731084_685875.html.
} 
tion de l'émergence et de la propagation de ces maladies sont également reconnus comme un bien public mondial $^{19}$ »(FAO et al., 2008). Et encore: «Puisque le potentiel d'une maladie reste inconnu jusqu'à ce qu'elle émerge, la surveillance des maladies infectieuses émergentes potentiellement pandémiques est clairement un bien public mondial ${ }^{20} \gg$ (FAO et al., 2008). C'est aussi l'action des Services vétérinaires nationaux et celle de l'OIE pour lesquelles sa directrice actuelle revendique un statut de bien public mondial (Éloit, 2012). Cette orientation s'inscrit concrètement dans les instruments internationaux de gestion de la santé animale dont témoignent, par exemple, la révision du code zoosanitaire de l'OIE et l'extension des obligations des États qui y sont associés (Figuié, 2014).

Pour les OI, la référence au bien public mondial doit permettre de mobiliser et de coordonner un réseau élargi et reconfiguré d'acteurs autour de l'objectif de biosécurité. L'opérationnalisation du concept de bien public mondial a cependant diverses limites. De façon générale, selon Gabas et Hugon (2001), les biens publics ne peuvent être les mêmes selon les sociétés, leur niveau de développement et leur insertion dans l'économie mondiale. Leur production suppose une coordination des États dans un contexte éventuel de conflits d'intérêts, de relations de pouvoir, de situation d'hégémonie et de dépendance. Certains auteurs avancent ainsi de façon critique que les biens publics mondiaux sont une forme de légitimation de l'action des OI, elles-mêmes dominées par les intérêts des pays occidentaux (Constantin, 2002).

Il faut en outre nuancer cette nouveauté de statut attribué à la santé animale. Le concept de BPM a d'abord été appliqué à la finance et au commerce international, et les premières dispositions internationales concernant la santé animale l'ont été dans un objectif de sécurisation du commerce international. Si les évolutions décrites ne sont pas totalement nouvelles, elles ont cependant pris une ampleur sans précédent. Le statut de BPM acquis par la santé animale, sans que sa définition n'en soit clarifiée, peut ainsi être mis au service d'objectifs non explicites divers, voire contradictoires. Dans la partie suivante, pour examiner les effets produits par l'élargissement du cadrage de la santé animale aux enjeux de biosécurité et la référence au bien public mondial, est exposé l'exemple de la grippe aviaire (H5N1) au Vietnam.

\footnotetext{
${ }^{19} \mathrm{Ma}$ traduction de 'Preventing emergence and cross-border spread of human and animal infectious diseases is considered to be a global public good... Surveillance systems that underpin the prevention of emergence and spread of such diseases are also recognized as a global public good'.

${ }^{20} \mathrm{Ma}$ traduction de 'as it is not clear what a disease's potential is until after it has emerged, surveillance for potentially pandemic EID is clearly a global public good'.
}

\section{Étude de cas : la grippe aviaire (H5N1)}

\section{La grippe aviaire au Vietnam : un risque local, une menace globale}

Le silence dans lequel continue aujourd'hui de circuler à l'échelle mondiale le virus $\mathrm{H} 5 \mathrm{~N} 1$ contraste avec le bouillonnement d'activités auquel les foyers épidémiques de 2003 ont donné lieu. L'apparition cette année-là d'un nouveau virus de grippe aviaire, le virus H5N1, a été l'occasion d'inscrire dans la pratique le concept de maladie émergente, de développer une approche biosécuritaire de la santé animale et de promouvoir une gouvernance zoosanitaire mondiale. À travers les outils mobilisés pour gérer la grippe aviaire au Vietnam, il est possible, en comparaison avec d'autres maladies, d'identifier la logique et les motivations qui ont sous-tendu sa gestion et son soutien par la communauté internationale.

La population vietnamienne est essentiellement rurale (à $70 \%$ ) et $90 \%$ des foyers élèvent des volailles. Lorsque la grippe aviaire (H5N1) a émergé, ces volailles, autoconsommées et vendues sur le marché national, assuraient $19 \%$ des revenus des ménages agricoles (Desvaux et Ton, 2008). Selon plusieurs experts, l'impact direct du virus sur les élevages est comparable à celui d'autres maladies aviaires classiques (comme la maladie de Newcastle [NCD] qui provoque régulièrement d'importantes mortalités chez les volailles, mais n'est pas transmissible aux humains). Pourtant, la NCD n'a jamais donné lieu à la même mobilisation que celle occasionnée par la grippe aviaire. L'enjeu local de santé publique, à lui seul, ne permet pas d'expliquer cette mobilisation. Le Vietnam n'a enregistré « que » 64 décès humains liés au virus $\mathrm{H} 5 \mathrm{~N}^{21}$ entre 2003 et 2014 , ce qui reste peu au regard d'autres maladies infectieuses qui affectent régulièrement le pays, en particulier la tuberculose $(12000$ décès dans le pays pour la seule année $2017^{22}$ ). Même l'augmentation subite et inexpliquée de $300 \%$ des cas de rougeole au Vietnam en 2014 n'a pas donné lieu à une mobilisation internationale, probablement du fait de l'absence de menace transfrontalière.

Dans le cas présent, ce ne sont pas les enjeux locaux ni la référence à un principe de solidarité qui ont principalement mobilisé la communauté internationale, mais la menace globale de pandémie et le souci d'agir de façon anticipée à la source, le Vietnam. Le virus H5N1 est associé à un ensemble de risques: risque de transmission d'animal à animal (risque épizootique), d'animaux à humains (risque zoonotique) et d'humains à

\footnotetext{
${ }^{21}$ www.who.int/influenza/human_animal_interface/ 20180921 tableH5N1.pdf.

${ }^{22}$ www.w.who.int/health-topics.
} 
humains (risque épidémique, voire pandémique si cette épidémie s'étend à une échelle mondiale). La plupart des cas humains enregistrés sont liés à des contacts répétés avec un animal vivant contaminé ou avec sa carcasse. Les transmissions interhumaines restent extrêmement rares.

Pour les spécialistes, l'évolution par mutation du virus $\mathrm{H} 5 \mathrm{~N} 1$ en un virus de grippe humaine facilement transmissible est inéluctable, sans que l'on puisse prévoir dans quel délai un tel événement se produira ni quelle sera la virulence du nouveau virus mutant. Et c'est pour faire face à ce futur virus pandémique que la communauté internationale s'est mobilisée de façon inédite, pour faire face donc à un virus qui «n'existait» pas encore (et «n'existe» toujours pas): le virus continue d'affecter les volailles (et occasionnellement les humains) mais il n'a pas muté en une forme transmissible directement d'humains à humains.

Il s'agit donc d'un danger incertain dans la mesure où la communauté scientifique ne connaît ni la probabilité d'une telle mutation (ignorance dont la communauté s'est affranchie en énonçant que le problème n'est pas de savoir «si » mais «quand»), ni la gravité (quelle sera la dangerosité $\mathrm{du}$ virus mutant transmissible entre humains?). Mais la référence aux effets dévastateurs de la pandémie de grippe de 1918 (la «grippe espagnole») a mobilisé les décideurs politiques et justifié une intervention rapide sur la base de la menace (risque incertain) et du principe de précaution.

La mobilisation de la communauté internationale pour la gestion de la grippe aviaire (H5N1) a été massive, le but étant de contenir la menace à sa source tant d'un point de vue géographique (l'Asie) que des espèces [les volailles] (Figuié, 2014). Ce cadrage adopté par les OI a guidé leurs interventions. La mobilisation de la FAO, de l'OMS et de l'OIE a été largement étudiée (Figuié, 2014; Zylberman, 2013; Brender et Gilbert, 2016); elle s'est organisée autour du principe forgé à l'occasion (même si l'idée sur laquelle il repose est ancienne), le principe «One World One Health» (FAO et al., 2008), qui deviendra par la suite «One Health».

Les premiers décès humains ont été enregistrés en 2003 en Chine et au Vietnam, faisant ainsi de ces pays aux yeux de la communauté internationale la ligne de front de la lutte contre le virus. La même année, un rapport commandé par le Congrès américain (CRS, 2006) souligne les nombreuses insuffisances dans la surveillance et le contrôle du virus dans les pays touchés. Ayant tiré les leçons des derniers attentats terroristes, le gouvernement américain comme celui d'autres pays occidentaux sont alors convaincus que pour leur propre sécurité, ils doivent augmenter leur participation à l'effort international et intervenir directement à la source des menaces, c'est-à-dire dans les pays touchés comme le Vietnam (Figuié, 2013).
La gestion du virus $\mathrm{H} 5 \mathrm{~N} 1$ affectant les volailles relève de la logique biosécuritaire et du processus de globalisation de la santé décrit plus haut: la menace d'une catastrophe pandémique jugée imminente a justifié la mobilisation d'importants dispositifs de surveillance et de contrôle du vivant (virus, animaux, humains). Les OI y ont occupé une place majeure, au nom de la santé globale, renforçant ainsi leur rôle dans la gestion d'affaires considérées jusque-là relevant du domaine national. C'est ainsi qu'a été créé le «Partenariat pour la gestion de la grippe aviaire et humaine/Partnership for Avian and Human Influenza (PAHI) » associant autorités vietnamiennes (ministères de la Santé, de l'Agriculture, du Commerce, des Finances, etc.) et OI (OMS, FAO, Unicef, Banque mondiale...). La mobilisation s'est faite par référence à l'intérêt général. Un représentant du ministère de la Santé déclarait ainsi : "L'abattage des volailles représente une perte économique importante pour les fermiers pauvres... mais le gouvernement est déterminé, et responsable à l'égard du reste du $\operatorname{monde}^{23}$ ». La rhétorique de l'intérêt général n'exclut pas des pressions très concrètes exercées par la communauté internationale, celle-ci ayant conditionné le succès de la demande d'adhésion du Vietnam à l'OMC à sa capacité de gestion du virus $(\mathrm{Vu}, 2009)$ et à prouver ainsi sa volonté de se montrer un bon citoyen du monde.

\section{La gestion locale de la grippe aviaire}

Trois éléments de la gestion de la grippe aviaire au Vietnam méritent d'être soulignés.

Le premier est relatif au recours des autorités publiques à des abattages sanitaires massifs de volailles. Durant les premiers mois de 2004, 17\% des volailles, soit 44 millions d'animaux (GSO, 2004), sont mortes, la plupart à la suite d'abattages préventifs. Les abattages sanitaires sont un mode courant de gestion des problèmes de santé animale. Un rapport de la Banque mondiale (2011) indique que pour l'ensemble des maladies animales dans le monde, $38 \%$ des pertes par décès d'animaux sont liées à des abattages préventifs (et que donc $62 \%$ des animaux meurent de la maladie); ce chiffre s'élève à $84 \%$ dans le cas de maladies zoonotiques. Dans les abattages sanitaires à visée d'éradication, les animaux sont assimilés à des marchandises pouvant être défectueuses ou pouvant

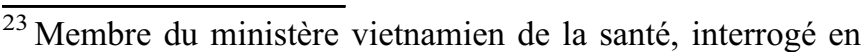
anglais le 17/12/2007. Ma traduction de 'This is a question which concerns not only this country, but all the world's countries. We must share this responsibility. Of course, the culling of chickens is a significant economic loss for the population, especially the poor, rural farmers... but the government is determined, and is also responsible to the rest of the world'.
} 
compromettre la valeur globale d'un lot de marchandises, et non comme des êtres sensibles.

Le deuxième élément est relatif à l'adoption par le gouvernement vietnamien de mesures visant, au nom de la biosécurité, à éliminer les «petits élevages » au profit d'une production industrielle jugée plus apte à adopter des pratiques de biosécurité et à servir le projet de modernisation agricole qui lui est étroitement associé (MARD et MOH, 2006). Ces mesures reposent d'une part sur une commodification accrue des animaux, et d'autre part sur l'éviction des éleveurs n'ayant pas les moyens d'adopter les normes de biosécurité. Cette dimension biosécuritaire de la santé animale est peu compatible avec les ambitions à court terme de développement rural et d'aide aux plus pauvres. Elle témoigne des contradictions portées par la mise en œuvre de la définition multidimensionnelle de la santé animale.

Le troisième élément est lié à la gestion de la grippe par les éleveurs vietnamiens. Ces derniers ont d'abord craint pour leur propre santé face à cette maladie que les autorités et les journaux ont, dès son apparition, comparée au Sras. Ils ont donc collaboré au dispositif national de surveillance mis en place par les autorités vietnamiennes en ligne avec les recommandations des OI (Figuié, 2013). Ils ont signalé aux autorités vétérinaires les foyers qui décimaient leurs élevages (et cela d'autant plus volontiers qu'ils espéraient une aide de ces autorités face à ce mal nouveau). Il y a donc eu dans un premier temps un alignement entre les cadres d'analyse (des autorités nationales, des éleveurs) pour considérer la grippe aviaire comme un risque pandémique, et une collaboration a donc été possible.

Mais au fil du temps, un désalignement s'est produit, particulièrement marqué dans les villages ayant expérimenté plusieurs foyers de grippe aviaire et où celle-ci est devenue endémique. Les éleveurs s'y sont familiarisés avec cette maladie et ont cessé de craindre pour leur propre santé. La menace pandémique s'est estompée à mesure que la comparaison au Sras s'avérait moins pertinente pour les éleveurs. Ces derniers ont révisé leur perception de la maladie et ont fini par l'appréhender comme une épizootie comparable à la maladie de Newcastle qui touche régulièrement leurs volailles (sans affecter les humains). Dans ce contexte, ils ont cessé de déclarer les cas aux autorités (et évité par la même occasion de potentiels abattages sanitaires), et le commerce illégal d'animaux malades ou provenant de zones affectées par la maladie s'est intensifié (Figuié et Desvaux, 2015).

Dans une autre étude sur la grippe aviaire au Vietnam, Porter (2012) montre un décalage entre la représentation de la grippe aviaire par les agents de santé et celle des éleveurs. Les premiers voient dans les volailles de simples marchandises, porteuses de virus dont les éleveurs à travers leurs (mauvaises) pratiques se font les vecteurs; ils préconisent des campagnes de sensibilisation visant à changer leurs comportements. Les seconds ont une approche socioécologique de la maladie: ils mettent en avant les interactions entre les volailles et les éléments naturels (vents, eaux, oiseaux sauvages...) pour expliquer la spatialisation de la maladie; ils privilégient des mesures de gestion respectueuses des normes sociales.

Cet exemple montre que le cadrage biosécuritaire conduit à élargir le domaine de la gestion de la santé animale pour inclure dans le champ du risque non plus seulement les maladies animales elles-mêmes, mais les animaux et les éleveurs. L'intervention publique s'est montrée peu intéressée à prendre en compte les savoirs locaux et les processus d'apprentissage collectif. Les éleveurs comme leurs animaux sont appréhendés comme des sources de risque à contrôler. Cette absence de reconnaissance et de légitimation des espaces profanes de gestion du risque entrave la possibilité d'une action collective cordonnée. Or, la gestion de l'incertitude associée aux menaces doit être nécessairement adaptive (au gré de l'évolution des connaissances permettant de réduire cette incertitude) et polycentrique (pour être capable de prendre en compte la diversité des contextes, des points de vue, des valeurs, des logiques et des intérêts...).

\section{Conclusion}

Depuis les premières politiques publiques de santé animale au XIX ${ }^{\mathrm{e}}$ siècle, leur champ d'intervention s'est considérablement élargi pour englober récemment des enjeux de biosécurité et en faire un bien public mondial. Cet élargissement est lié à l'importance prise par les maladies infectieuses émergentes zoonotiques dans l'agenda sanitaire des OI. Il accentue le caractère multidimensionnel mais aussi ambigu de la santé animale. À travers cet objet et son histoire se lit la façon dont les sociétés abordent les risques liés au vivant, les relations entre humains et animaux, mais aussi les relations internationales.

Les politiques de santé animale ont une approche anthropocentrée de l'animal, que les "animal studies» n'ont que marginalement analysée (Cassidy et al., 2017). La montée en puissance d'une «emerging disease worldview» (King, 2002) étroitement associée aux zoonoses s'oppose à l'acquisition pour les animaux d'un statut de victime, et en fait une source inépuisable de risques. L'étendue du domaine d'action de la santé animale, l'ambition de prendre en charge les menaces (en opposition aux seuls risques avérés) conduisent à attirer dans le champ d'intervention de ces politiques non seulement les animaux "souffrants 》 mais aussi ceux jugés peu productifs, hors normes, les réservoirs d'agents 
pathogènes ou potentiellement pathogènes (comme les chauves-souris), autrement dits les animaux «malades des Hommes » pour reprendre l'expression de Keck (2012). La façon dont l'évolution de ces politiques, historiquement centrées sur les animaux d'élevage, remet en jeu les termes du contrat domestique (Larrère et Larrère, 1997) et intègre davantage dans leurs interventions les animaux sauvages (à la faveur de l'adoption du concept One Health) mériterait de plus amples développements.

Dans un contexte de globalisation de la santé, les pays du Sud sont perçus comme des sources majeures de menaces. Ces pays sont désignés comme le «réacteur» de l'émergence de nouvelles maladies du fait de leur biodiversité, de la proximité humains-animaux dans les zones de haute densité démographique. De plus, la faiblesse des services de santé y réduit la capacité de contrôle des maladies. La géographie du sous-développement recouvre en bonne partie celle des émergences et des menaces qui leur sont associée. Cette menace n'a pas besoin de se concrétiser: il suffit d'avoir l'autorité de l'énoncer pour produire des effets déstabilisateurs sur les sociétés, sur la circulation des personnes et des biens, circulation dont la garantie est à la base du principe moderne de sécurité (Gros, 2012).

Le concept englobant de bien public mondial tend à masquer la diversité des intérêts en jeu. Il permet d'enrôler les États au service d'un intérêt supposé a priori commun et agit comme un processus de légitimation de l'action des organisations internationales. Le cadrage biosécuritaire monte en puissance sans que soit ouvert le débat sur ce qu'il peut porter de renoncement aux objectifs et aux principes qui guident les autres dimensions de la santé, et de la santé animale en particulier.

La santé animale est un objet politique qu'il faut dénaturaliser. Notre contribution vise à en montrer les différentes dimensions et à clarifier les enjeux associés. Les sciences sociales peuvent permettre de comprendre les intérêts, les valeurs et leur mise en concurrence discrète dans le concept de santé animale et contribuer ainsi à ouvrir un débat sur ce que nous voulons en faire pour construire un monde plus sûr mais aussi plus solidaire entre États comme entre humains et animaux.

\section{Remerciements}

Je remercie François Buton, Bernard Hubert, mes collègues du groupe de travail Humains/Animaux, Martine Antona, Sigrid Aubert, Céline Dutilly et Nicolas Gaidet, ainsi que les relecteurs pour leurs commentaires précieux sur les versions préliminaires de ce texte (dont le contenu n'engage que son auteur).

\section{Références}

Banque mondiale, 2011. World livestock disease atlas: a quantitative analysis of global animal health data (2006-2009), Washington (DC), World Bank, http://hdl. handle.net/10986/27118.

Barbier M., 2002. La gouvernance des risques alimentaires et la société du risque. Une approche à partir de la «saga de la vache folle », Cahier du GEMDEV, 28, 21-41.

Barroux G., 2011. La santé des animaux et l'émergence d'une médecine vétérinaire au XVIII ${ }^{\mathrm{e}}$ siècle, Revue d'histoire des sciences, 64, 349-376, https://www.jstor.org/stable/ 23634474.

Beck U., 2001 ( $1^{\mathrm{re}}$ éd. 1986). La société du risque. Sur la voie d'une autre modernité, Paris, Aubier. Traduit de: Risikogesellschaft. Auf dem Weg in eine andere Moderne, Frankfurt am Main, Suhrkamp, 1986.

Beck U., 2009. World at Risk, Cambridge, Polity Press.

Berdah D., 2010. La vaccination des bovidés contre la tuberculose en France, 1921-1963: entre modèle épistémique et alternative à l'abattage, Revue d'Études en Agriculture et Environnement, 91, 4, 393-415.

Bingham N., Enticott G., Hinchliffe S., 2008. Biosecurity: spaces, practices, and boundaries, Environment and Planning A, 40, 1528-1533, https:/www.doi.org/10.1068/ a4173.

Boidin B., 2014. La santé, bien public mondial ou bien marchand? Réflexions à partir des expériences africaines, Villeneuve-d'Ascq, Presses universitaires du Septentrion.

Bonnaud L., Fortané N., 2016. Au-delà des crises de santé animale. Pour une sociologie de l'action publique vétérinaire, Gouvernement et Action Publique, 5, 3, 131140, https://doi.org/10.3917/gap.163.0131.

Bonnaud L., Fortané N., 2018. L'État sanitaire de la profession vétérinaire. Action publique et régulation de l'activité professionnelle, Sociologie, 9, 3, 253-268, http://journals. openedition.org/sociologie/3502.

Bonnaud L., Fortané N., 2021a. 21st century vets: professional dynamics in the era of One Health, Review of Agricultural, Food and Environmental Studies, 102, 121-124, https://doi. org/10.1007/s41130-021-00141-3.

Bonnaud L., Fortané N., 2021b. Being a vet: the veterinary profession in social science research, Review of Agricultural, Food and Environmental Studies, 102, 125-149, https:// doi.org/10.1007/s41130-020-00103-1.

Bourg D., Joly P.-B., Kaufmann A. (Eds), 2013. Du risque à la menace. Penser la catastrophe, Paris, Presses universitaires de France.

Brender N., Gilbert C., 2016. De l'émergence aux émergences. Le cas de la pandémie grippale, in Morand S., Figuié M. (Eds), Émergence de maladies infectieuses. Risques et enjeux de société, Versailles, Quæ, 37-62.

Brown K., Gilfoyle D., 2010. Healing the herds: disease, livestock economies and the globalization of veterinary medecine, Athens (Ohio), Ohio University Press.

Brown T.M., Cueto M., Fee E., 2006. The World Health Organization and the transition from "international" to 
"global" public health, American Journal of Public Health, 96, 1, 62-72, https://doi.org/10.2105/AJPH.2004.050831.

Buton F., 2008. Sous l'empire des risques sanitaires : les métamorphoses de la santé publique, Savoir/Agir, 5, 3, 7582, https://doi.org/10.3917/sava.005.0075.

Calain P., 2007. Exploring the international arena of global public health surveillance, Health, Policy and Planning, 22, 2, 2-12.

Callon M., Lascoumes P., Barthe Y., 2001. Agir dans un monde incertain. Essai sur la démocratie technique, Paris, Le Seuil.

Cassidy A., Dentinger R.M., Schoefert K., Woods A., 2017. Animal roles and traces in the history of medicine, c. 1880-1980, The British Journal for the History of Science, 2, https://doi.org/10.1017/bjt.2017.3.

Chien Y.J., 2013. How did international agencies perceive the avian influenza problem? The adoption and manufacture of the 'One World, One Health' framework, Sociology of Health \& Illness, 35, 2, 213-226, https://doi.org/10.1111/ j.1467-9566.2012.01534.x.

CIA (Central Intelligence Agency), 2003. SARS: lessons from the first epidemic of the 21st century. A collaborative analysis with outside experts. Unclassified report, Washington (DC), CIA, www.hsdl.org/?view\&did=481435.

CRS (Congressional Research Service), 2006. U.S. and international responses to the global spread of avian flu: issues for Congress, Washington (DC), Congressional Research Service, https://sgp.fas.org/crs/misc/RL33219. pdf.

CCE (Commission des communautés européennes), 2007. Une nouvelle stratégie de santé animale pour l'Union européenne (2007-2013) placée sous la devise "Mieux vaut prévenir que guérir ». Communication de la Commission au parlement européen, au conseil économique et social européen et au comité des régions, Luxembourg, Office des publications officielles des communautés européennes, https://eur-lex.europa.eu/legal-content/FR/TXT/PDF/?uri= CELEX:52007DC0539\& from=FR.

Constantin F. (Ed.), 2002. Les biens publics mondiaux. Un mythe légitimateur pour l'action collective?, Paris, L'Harmattan.

Dean M., 1999. Risk, calculable and incalculable, in Lupton D. (Ed.), Risk and sociocultural theory. New directions and perspectives, Cambridge, Cambridge University Press, 6784.

Demortain D., 2019. Une société (de l'analyse) du risque ?, Natures Sciences Sociétés, 27, 4, 390-398, https://doi.org/ 10.1051/nss/2020005.

Desvaux S., Ton V.D. (Eds), 2008. A general review and a description of the poultry production in Vietnam, Hanoi, Agricultural Publishing House.

Elias N., 1973. La civilisation des moeurs, Paris, CalmannLévy.

Éloit M., 2012. Le concept de bien public mondial, promoteur d'une bonne gouvernance vétérinaire, Revue scientifique et technique de l'Office international des épizooties, 31, 2, 577-584.
Enticott G., 2012. The local universality of veterinary expertise and the geography of animal disease, Transactions of the Institute of British Geographers, 37, 1, 75-88, https://doi. org/10.1111/j.1475-5661.2011.00452.x.

Ewald F., 1986. L'État providence, Paris, Grasset.

FAO (Food and Agriculture Organization), 2013. World Livestock 2013. Changing disease landscape, Rome, FAO.

FAO, 2008. Contributing to One World, One Health. A strategic framework for reducing risks of infectious diseases at the animal-human-ecosystems interface, $\mathrm{FAO} /$ OIE/World Health Organization, www.preventionweb.net/ files/8627_OWOH14Oct08.pdf.

Fidler D., 2003. SARS: political pathology of the first postwestphalian pathogen, Journal of Law, Medicine \& Ethics, 31, 4, 485-505, https://doi.org/10.1111/j.1748-720X.2003. tb00117.x.

Figuié M., 2013. Global health risks and cosmopolitisation: from emergence to interference, Sociology of Health \& Illness, 35, 2, 227-240, https://doi.org/10.1111/ j.1467-9566.2012.01539.x.

Figuié M., 2014. Towards a global governance of risks: international health organisations and the surveillance of emerging infectious diseases, Journal of Risk Research, 17, 4, 469-483, https://doi.org/10.1080/ 13669877.2012.761277.

Figuié M., Pham A.T., Moustier P., 2013. Grippe aviaire dans la filière. La réorganisation du secteur agro-industriel au Vietnam, Revue d'Études en Agriculture et Environnement, 94, 4, 397-419, https://doi.org/10.22004/ag.econ.196605.

Figuié M., Desvaux S., 2015. Managing global risks: Vietnamese poultry farmers and avian flu, in Morand S., Dujardin J.-P., Lefait-Robin R., Apiwathnasorn C. (Eds), Socio-ecological dimensions of infectious diseases in Southeast Asia, Singapore, Springer, 257-273.

Fortané N., Keck F., 2015. Ce que fait la biosécurité à la surveillance des animaux, Revue d'anthropologie des connaissances, 9, 2, 125-137, https://doi.org/10.3917/ rac.027.0125.

Foucault M., 2004. Sécurité, territoire, population. Cours au Collège de France, 1977-1978, Paris, Gallimard/EHESS/ Seuil.

Gabas J.-J., Hugon P., 2001. Les biens publics mondiaux et la coopération internationale, L'Économie politique, 12, 4, 1931, https://doi.org/10.3917/leco.012.0019.

Gardon S., Gautier A., Le Naour G., 2019. La santé globale au prisme de l'analyse des politiques publiques, Marcyl'Étoile, Éditions de l'École nationale des services vétérinaires VetAgro Sup.

Gilbert C., 2002. La fin des risques?, Quaderni, 48, 111-120, https://www.persee.fr/doc/quad_0987-1381_2002_ num 48 1_1748? $\mathrm{q}=\mathrm{la}+\mathrm{fin}+\mathrm{des}+$ risques.

Gilbert $\bar{C}$. (Ed.), 2003. Risques collectifs et situations de crise. Apports de la recherche en sciences humaines et sociales, Paris, L'Harmattan.

Godard O., Henry C., Lagadec P., 2002. Traité des nouveaux risques. Précaution, crise, assurance, Paris, Gallimard.

Gros F., 2012. Le principe sécurité, Paris, Gallimard. 
GSO (General Statistics Office of Vietnam), 2004. Socioeconomic impact of Avian influenza, Hanoi, GSO.

Hubscher R., 1999. Les maîtres des bêtes. Les vétérinaires dans la société française, XVIII ${ }^{e}$-XXe siècle, Paris, Odile Jacob.

Jerolmack C., 2013. Who's worried about turkeys? How 'organisational silos' impede zoonotic disease surveillance, Sociology of Health \& Illness. Special Issue: Pandemics and emerging infectious diseases: the sociological agenda, 35, 2, 200-212, https://doi.org/10.1111/ j.1467-9566.2012.01501.x.

Jones K.E., Patel N.G., Levy M.A., Storeygard A., Balk D., Gittleman J.L., Daszak P., 2008. Global trends in emerging infectious diseases, Nature, 451, 990-993, https://doi.org/ 10.1038/nature06536.

Joshi N.P., Herdt T.H. (Eds), 2006. Production diseases in farm animals, Wageningen, Wageningen Academic Publishers.

Keck F., 2012. Anthropologie sociale et maladies animales, Cahiers d'anthropologie sociale, 8, 1, 11-24, https://doi. org/10.3917/cas.008.0011.

Kerouedan D., 2013. Géopolitique de la santé mondiale, Paris, Collège de France/Fayard.

Kindleberger C.P., 1988. The international economic order. Essays on financial crisis and international public goods, New York, Harvester Wheatsheaf.

King N.B., 2002. Security, disease, commerce: ideologies of postcolonial global health, Social Studies of Sciences, 32, 5-6, 763-789, https://doi.org/10.1177/030631270203200507.

King N.B., 2004. The scale politics of emerging diseases, Osiris, 19, 1, 62-76.

Lagadec P., 1981. La civilisation du risque. Catastrophes technologiques et responsabilité sociale, Paris, Seuil.

Lakoff A., Collier S.J. (Eds), 2008. Biosecurity interventions. Global health and security in question, New York, Columbia University Press.

Landais E., 1990. Sur les doctrines des vétérinaires coloniaux français en Afrique noire, Cahiers des Sciences Humaines, 26, 1-2, 33-71.

Larrère C., Larrère R., 1997. Le contrat domestique, Le Courrier de l'environnement de l'INRA, 30, 5-19.

Law J., Mol A., 2011. Veterinary realities: what is foot and mouth disease?, Sociologia Ruralis, 51, 1, 1-16, https://doi. org/10.1111/j.1467-9523.2010.00520.x.

MARD (Ministry of Agriculture and Rural Development, Vietnam), MOH (Ministry of Health, Vietnam), 2006. Integrated national operational program for the avian and human influenza (OPI), 2006-2010, (Green Book), Hanoi, MARD, MOH.

Michalon J., 2019. One health au prisme des sciences sociales : quelques pistes de lecture, Bulletin de l'Académie Vétérinaire de France, 172, 1, 118-122, https://doi.org/ 10.4267/2042/70672.
Morand S., 2016. Biogéographie et écologie de l'émergence, in Morand S., Figuié M. (Eds), Émergence de maladies infectieuses. Risques et enjeux de société, Versailles, Quæ, 13-36.

OMS (Organisation mondiale de la santé), 2007. The world health report 2007. A safer future: global public health security in the 21st century, Genève, OMS.

Porter N., 2012. Risky zoographies: the limits of place in avian flu management, Environmental Humanities, 1, 1, 103-121, https://doi.org/10.1215/22011919-3609994.

Smith R., Woodward D., Acharya A., Beaglehole R., Drager N., 2004. Communicable disease control: a 'global public good' perspective, Health Policy and Planning, 19, 5, 271278, https://doi.org/10.1093/heapol/czh032.

Stanziani A., 2005. Histoire de la qualité alimentaire. XIX ${ }^{e}-$ $X X^{e}$ siècle, Paris, Seuil.

Swabe J., 1999. Animals, disease and human society. Humananimal relations and the rise of veterinary medicine, London, Routledge.

Taylor-Gooby P., Zinn J.O., 2006. Current directions in risk research: new developments in psychology and sociology, Risk Analysis, 26, 2, 397-411, https://doi.org/10.1111/ j.1539-6924.2006.00746.x.

Teissier M., 2007. Une brève histoire de l'OIE, Bulletin de l'OIE, 1, 6-18.

Vagneron F., 2015. Surveiller et s'unir? Le rôle de l'OMS dans les premières mobilisations internationales autour d'un réservoir animal de la grippe, Revue d'anthropologie des connaissances, 9, 2, 139-162, https://doi.org/10.3917/rac.027.0139.

Vallat B., 2007. Éditorial. Protéger notre planète des maladies émergentes liées à la mondialisation, Bulletin de l'OIE , 2, 1-3.

Vu T., 2009. The political economy of avian influenza response and control in Vietnam, Brighton, STEPS Working Paper, 19.

Woods A., 2004. The construction of an animal plague: foot and mouth disease in nineteenth-century Britain, Social History of Medecine, 17, 1, 23-39, https://doi.org/10.1093/ $\mathrm{shm} / 17.1 .23$.

Woods A., Bresalier M., 2014. One health, many histories, Veterinary Record, 174, 26, 650-654, https://doi.org/ 10.1136/vr.g3678.

Wynne B., 1996. May the sheep safely graze? A refelexive view of the expert-lay knowledge divide, in Lash E., Szerszynski B., Wynne B. (Eds), Risk, environment and modernity: towards a new ecology, London, Sage, 44-83.

Zinsstag J., Schelling E., Waltner-Toews D., Tanner M., 2011. From "one medicine" to "one health" and systemic approaches to health and well-being, Preventive Veterinary Medicine, 101, 3-4, 148-156, https://doi.org/10.1016/j. prevetmed.2010.07.003.

Zylberman P., 2013. Tempêtes microbiennes. Essai sur la politique de sécurité sanitaire dans le monde transatlantique, Paris, Gallimard.

Citation de l'article: Figuié M. La gouvernance de la santé animale : entre biosécurité et bien public mondial. Nat. Sci. Soc. 29, $3,274-287$. 